\title{
The Need of Using Technology in Mathematics
}

\author{
${ }^{1 .}$ Miss. Sandhya V. Keche
}

\begin{abstract}
The use of technology has a long history in mathematics education. Many societies, for example, introduce arithmetic with an abacus, for two reasons. First, the abacus supports computation. Second, the abacus presents a tangible image of mathematics, which helps students understand difficult concepts. Technology can reduce the effort devoted to tedious computations and increase students' focus on more important mathematics. Equally importantly, technology can represent math in ways that help students understand concepts. In combination, these features can enable teachers to improve both how and what students learn.
\end{abstract}

\section{Introduction}

All people face limits in the number of levels or details they can keep track of during problem solving. In addition to the unavoidable difficulty of a particular math problem, learners may experience additional cognitive load (i.e., thinking difficulties) in the materials and tools they use. Teachers should minimize load that is unimportant to the current learning goal and direct student activity to thinking that is germane to what they should be learning (Sweller, 1988). Technology can be useful to the extent it focuses student thinking in ways that are germane, not extraneous.

What is important or germane depends on the math topic and age of the student. In primary school, it is important to learn to do arithmetic fluently. Using technology to do this thinking for the student would be inappropriate. In secondary school, however, students have mastered arithmetic and should be focused on more advanced skills and concepts. Computational support for lower-order details can then be very important. researchers have found that when calculators are available to offload details computations, teachers can better focus on

- More realistic or important problems.

- Development of flexible strategies.

- Mathematical meaning and concepts.

- $\quad$ Exploration \& Sense-Making

with multipresentations.

Before the development of the computer, we therefore had various forms of mathematics, including:

1. Enactive mathematics, with physical actions on actual objects,

2. Visual mathematics described verbally, with physical properties of objects verbalised and built into a systematic deductive theory as in Euclidean geometry,

3. Symbolic mathematics, (arith-metic, algebra, calculus etc.), arising from actions on real-world objects (such as counting)and developing through computation and symbol manipulation,

4. A combination of (2) and (3) linking symbolism and graphical representation,

5. Formal mathematics, with concepts definedby verbal-symbolic axiomsand further properties deducedby formal proof.

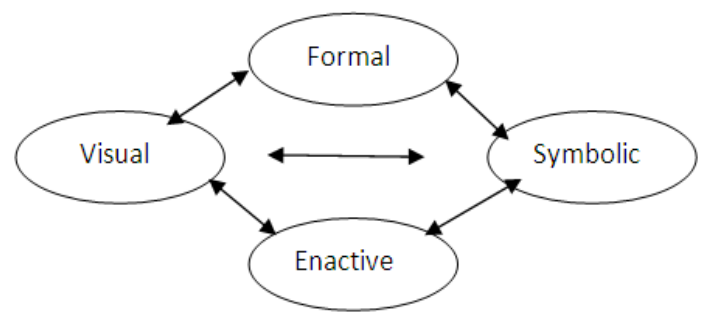

II. What Technology Can Do For Education

The most useful educational perspective on information technologies is to see them primarily as tools that help students accomplish more. The tool role is of primary importance, because it extends the capacity of students to undertake investigations, to attack computational problems, to communicate, and to access 
information resources. Secondarily, information technologies also can be used for interesting simulations students can learn about through exploration and interaction.

Information technologies offer new ways of structuring the curriculum to both support student inquiry and cover more content. A major commitment to using technological tools across the curriculum will permit more advanced mathematics and science concepts to be treated. Computational power frees mathematics students to explore both mathematical and real-world phenomena where in the past the computational complexity would have been a barrier. Computers can also mount brute force attacks on problems that previously required abstract formalisms far beyond the reach of most precollege students. With this power, students can investigate and understand topics that are beyond the current curriculum standards. In the long run, the tool and simulation capacities of information technologies can have four different levels of impacts on the curriculum:

\section{Level 1. Substitution.}

At the first level, information technologies are used to accomplish the existing curriculum goals but doing them better or to a higher level of student comprehension. For instance, labs based on probes connected to computers have the ability to reach very high levels of student comprehension of material previously mastered by few students.

\section{Level 2. Addition.}

At this level, technology makes it possible to achieve new curriculum goals, usually by adding new material to an existing course. For instance, the TERC Global Lab project adds to existing science courses the possibility of international collaboration and peer review by creating groups of schools linked through telecommunications.

\section{Level 3. Disciplinary restructuring.}

At this level, the capacity of information technologies makes it possible to redesign a course or series of courses within a discipline. For instance, graphing can be introduced far earlier in the math sequence giving students skills that can help speed their understanding of many concepts that might have a graphical interpretation.

\section{Level 4. Interdisciplinary restructuring.}

At this level, technology supports the redesign of courses across disciplines. For instance, if systems modeling was learned in ninth grade math, then subsequent science courses could use this capacity to address a broader range of science material at a deeper level. These four levels involve increasing difficulty and educational payoff. Clearly, the first level is simplest to implement, because it is easy to substitute an improved, technology-based approach to a topic for a less effective one. This strategy has given rise to tens of thousands of small computer programs, far more than can be addressed in this article. The ease of implementation has often outweighed considerations of quality; just because the material is treated with technology does not guarantee that the learning is better.

Level two is more difficult to implement because curricula represent a zero-sum game: for every topic added, something must be dropped. Making curriculum changes and justifying them to all the concerned educators and parents takes time and effort. Still, there are many cases in which educators undertake this effort, understanding the importance that the new capacity of technology adds. Levels three and four represent largescale course changes that are largely untried and unstudied. Yet, these kinds of change promise the greatest rewards, providing students with far deeper understanding of much more content that is currently expected. Such large-scale changes are difficult for a single school or school district to implement, because their graduates might not have the familiar set of knowledge and because transfer students, in and out of the system, will face severe difficulties. As a result, such really large-scale change must await regional, state, or national consensus that is very difficult to achieve. It is important, therefore, to begin our survey with the emerging national consensusabout what curricula should be and what the role of information technology might be. The recentlypromulgated national standards for mathematics and science are probably the most thoughtful compendia of what is needed, and so we begin by

\section{Important Packages}

In this section, three packages will be discussed: ClarisWorks, Microsoft Works, and Alice. All are widely used in schools and all run on both the Macintosh andWintel computer platforms. This covers all the computers schools are currently purchasing. Schools with a large backlog of older computers need not despair, however, since perfectly good productivity packages are available for every computer ever made and the skills learned with one package are easily transferred to others. ClarisWorks is a productivity package that many 
schools have adopted.It is inexpensive and limited but provides adequate performance for many school applications and is easy to learn. Most importantly, it is easy to create compound documents within ClarisWorks. This means, for instance, that a spreadsheet can be embedded in a formatted text document that also includes drawings, databases, and other objects. The embedded spreadsheet is not just a picture of the state of spreadsheet, but a live application that can be opened, used, modified, and saved.This supports collaboration both within a class and over networks.Microsoft Works is a close second as an inexpensive productivity package. But, to get the best general spreadsheet, you a better choice is the Microsoft Excel spreadsheet . It has more options, more cells, more functions, and all kinds of formatting features. The latest version of Excel has the BASIC programming language just under the surface, so when students need additional horsepower in their spreadsheets, they are ready to learn some new programming concepts. It is important to teach programming, and a problem students need to solve can provide the context and motivation for learning this skill. Alice is a software package that shares some features with productivity packages, but was developed specifically for schools. Alice was designed to simplify sharing data over networks and was kept simple to use in order to make it accessible to beginning users. It has tables that are a bit like spreadsheets but donot have different calculations for each cell; instead, its calculations are done on entire columns. While this limits its flexibility, having the same rule for every number in a column is clearer and more intuitive for neophytes. It also makes it easy to use built-indatabase functions since each row can be treated as a record. Alice's graphing capacity is better in some important respects than most spreadsheets, and if the datainclude latitude and longitude columns, they can be plotted on a zoom-able map. Innetworking applications, Alice table data can be sent off to a database where it is combined with data from others and downloaded. This is ideal for collecting and sharing environmental data collected by students. Best of all, Alice is free. A system-wide strategy for spreadsheets might start students in early elementary grades simply using spreadsheets to explore patterns such as indicated in the addition table example. Ironically, an advanced spreadsheet like Excel might be best for this level because applications built from it can have a user interface that is easier to use and relatively bullet-proof. In the upper elementary grades, students will be ready to go "behind" the user interface and need the simplest package. Alice might be a good choice here, particularly if it were introduced in the context of an environmental data sharing project. Middle grade students might begin using a simple spreadsheet like ClarisWorks in both mathematics and science while learning its other applications in arts, communications, and social science classes. At this level, student projects could begin to exploit the power of these tools. By high school, students might need to graduate to the additional power of Excel that would be used extensively in mathematics, cience, technology, and social science cources.

\section{Other Tool Software}

While spreadsheets are underrated and should be central to any school's information technology strategy, they cannot do everything, and there are additional software tools that should be part of any precollege mathematics and science program. This is especially true in biology, where spreadsheets prove a less felicitous match for methods and explorations typical of the life sciences

In the following, seven additional kinds of more specific applications will be recommended each of which represents an important kind computational capacity.The educational justification for each of these is the same as that for spreadsheets:students need to master powerful general tools that can be applied to their own investigations, and they need a basic understanding of the capacities of this technology that is transforming so much of science, technology, and society MBL is an important counterbalance to the tendency of information technologies to divorce learning from reality. Although simulations are available for every common teaching lab, they can never be as good as the real thing. Simulations have their role for labs that cannot be offered for reasons of safety, scale, or cost, but every effort must be made to increase the amount of exposure students have to real experimental situations. MBL harnesses the power of information technologies to make labs a better learning environment and to increase the range of investigations students can undertake.

\section{Databases, Statistics, and Tabletop}

Graphing software and databases are, in many respects, complementary. Like graphers, databases are tools that help students build theories \& understand observations and are particularly important when there are lots of observations. Databases are particularly useful when the data are categorical, when there are many possible variables only some of which might be related, and when statistics is important due to high variability in the data. Databases are under-utilized in schools, in part because the fear so many has of statistics. There are software tools that help students understand categorical data and make inferences without needing formal statistics even when the data has considerable random variability. Perhaps the most general and accessible are Tabletop and Tabletop Junior. In Tabletop, each data record is represented by an icon that can be interrogated by clicking. The icons can be sorted by the values of their variables using logical functions or axes. As the sorting conditions change, the icons move smoothly into their new positions. The results are appealing and very 
revealing. Scatter diagrams, Venn diagrams, and cross-tabulations all seem quite natural and comprehensible in this environment

\section{Information Technologies \& Instructional Strategies}

Information technologies must be embedded in excellent curricula that takea dvantage of the new opportunities for learning created by the technology. We have seen a well-tested explorative MBL lab rendered meaningless to students by a teacher who could not foster student exploration. Marcia Linn has conclusively demonstrated the role of curriculum in a remarkable series of classroom experiments. Starting with $3 \%$ of the students able to distinguish accurately between heat and temperature, students reached only $10 \%$ using the first curriculum. But after eight iterations with the same apparatus, they reached 50\%. In over a decade of research and refinement of the hardware, software, and curriculum, Ron Thornton has shown that exploration with MBL can improve student qualitative understanding of mechanics dramatically better than any combination of traditional labs, lectures, and homework (see, for instance, Thornton and Sokoloff, 1990). Then, using interactive MBL demonstrations with a large display that a whole class can see, combined with good instructional strategies thatengage every student, he can achieve a stable increase in understanding that verges on complete comprehension for all students (Thornton, 1996). In both these cases, MBL technology was necessary but not sufficient to achieve the educational goals; excellent curricula, consisting of quality instructional strategies,materials, and learning goals, were equally necessary. It is the importance of excellent curricula and the difficulty of developing it that needs to be underscored.Too many technology advocates assume that access to information technologies will drive reform; "Just give them access to the Internet" is their rallying call. But it is never that simple. These examples demonstrate how much effort is required to craft an excellent curriculum; that multiple cycles of in-class testing and revision are needed before the greatest learning is achieved. Unfortunately, there is little curriculum material that takes good advantage of technology and is available to schools.

\section{The Need for More IT-Rich Curricula}

It is no accident that the Voyages and the Kids Network are excellent, because they were carefully developed over five to ten years by highly creative teams of experts. They each required multiple iterations of testing and revision in a wide range classrooms; the Kids Network was tested in 200 classrooms. The total development cost of each curriculum was between five to ten million dollars. In terms of a single school's budget or other educational projects, these are huge expenses; they are, however, comparable to what a publisher invests in a new text or series of texts, and infinitesimal compared to the billions invested nation-wide in texts, computers,or sports equipment.

What is tragic is that both these projects were designed over a decade ago and that there are no comparable projects underway that fully exploit the substantial curriculum advances possible with the better technology now available. Very few technology-driven projects are able to invest the level of funding available for curriculum development of the Kids Network and the Voyage of the Mimi. As a direct result, there are very few excellent technology-rich curricula. In fact, the high cost of developing curricula leads curriculum developers away from information technologies, because they want to reach the largest number of students possible. Placing any restrictions on who can use the new curriculum, such as they must have Internet access or use MBL, runs counter to the desire to reach every student. As a consequence, a gulf has opened between people creating new curricula and those advocating information technologies; the curriculum developers are shy of technology and the technology promoters are forced to downplay the importance of curricula and advocate doing without. Until there is adequate recognition of this problem by funders able to cover the high costs of developing technology-rich curricula, new projects will tend to either ignore information technologies or treat them as optional add-ons. Unfortunately, this confines most new material to level one (substitution) or two (addition), making it impossible to find fundamentally new courses based on information technology.

It is important to note that radical changes of the sort described above are largely untried. Several of the ideas are supported by research (for instance students can build dynamic models from spreadsheets at least as early as ninth grade) but no individual schools have undertaken such major revisions (Tinker, 1990). There are many reasons for the absence of significant case studies:

Risk. The fact that these more radical changes are untried makes them difficult to justify to parents and educators.

Scale. Such changes would need to be undertaken across an entire district and over many years.

Costs. The development of good curriculum is expensive, and this would require a great deal of new material.

Population shifts. Once in place, students transferring into and out of the system would be at a great disadvantage. 
Staff knowledge. Teacher preparation at all levels is so thin in mathematics and science that few teachers are able to adopt more advanced content without substantial inservice support.

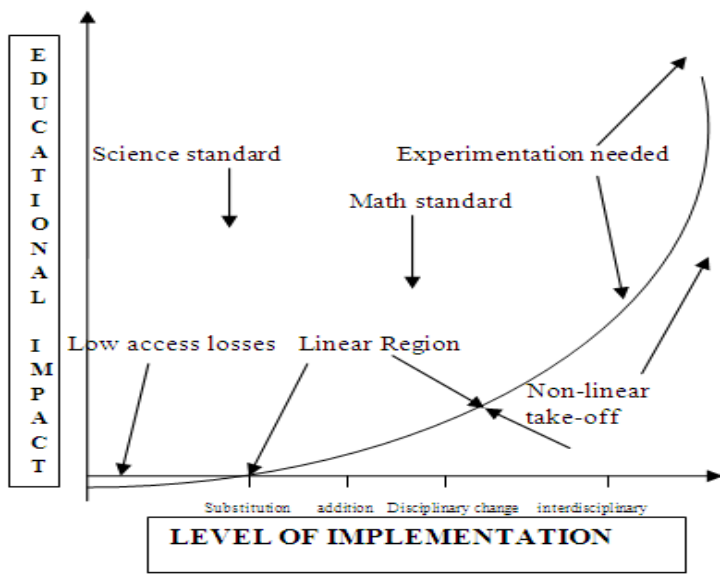

\section{References}

[1]. Bikner-Ahsbahs, A., \& Prediger, S. (2010). Networking of theories - an approach for exploiting the diversity of theoretical approaches. In B. Sriraman \& L. English (Eds.), Theories of mathematics education: seeking new frontiers (pp.483506). New York: Springer.

[2] Drijvers, P., Doorman, M., Boon, P., Reed, H., \& Gravemeijer, K. (2010). The teacher and the tool; instrumental orchestrations in the technology-rich mathematics classroom. Educational Studies in Mathematics, 75(2), 213-234.

[3] Hoyles, C., \& Lagrange, J.-B. (Eds.)(2010). Mathematics education and technology -Rethinking the terrain. NewYork/Berlin:Springer.

[4] Kaput, J. (2007). Technology becoming infrastructural in mathematics education. Models \& Modeling as Foundations for the Future in Mathematics Education. Mahwah, NJ: Lawrence Erlbaum.

[5] Roschelle, J., Tatar, D. , Shechtman, N., Hegedus, S., Hopkins, B., Knudsen, J., Stroter, A. (2007). Can a Technology-enhanced Curriculum Improve Student Learning of Important Mathematics? Menlo Park, CA: SRI International. Available at: http://math.sri.com/publications/index.html.

[6] Fuglestad, A.B. (2007). Teaching and teachers' competence with ICT in mathematics in a community of inquiry. Proceedings of the 31 st Conference of the International Group for the Psychology of Mathematics Education (pp. 2-249 - 2-258). Seoul, Korea.

[7] Kieran, C., \& Drijvers, P. (2012). The didactical triad of theoretical framework, mathematical topic, and digital tool in research on learning and teaching. Paper presented at the Colloque Hommage à Michèle Artigue, Paris, May, 31st, 2012.

[8] Ruthven, K. (2007). Teachers, technologies and the structures of schooling. In Pitta-Pantazi,D., \& Philippou, G., Proceedings of the V Congress of the European Society for Research in Mathematics Education CERME5 (pp. 52-67). Larnaca, Cyprus: University of Cyprus.

[9] Pea, R. (1987). Cognitive technologies for mathematics education. In A.H. Schoenfeld (Ed.),Cognitive Science and Mathematics Education (pp. 89-122). Hillsdale, NJ: Lawrence Erlbaum.

[10] Monaghan, J. (2005). Computer Algebra, instrumentation and the Anthropological Approach. Paper Presented at the 4th CAME onference, October 2005.

http://www.lonklab.ac.uk/came/events/CAME4/index.html. Accessed April 7, 2012. 